\title{
Evaluation of a Therapy Dog in General Adult Psychiatry Outpatient Clinic
}

\author{
Dr Rebecca Crease, Dr George Hunton, Dr Adam Joiner
}

\section{ABSTRACT}

Recent studies on inpatient psychiatric populations have found significant benefits of 'pet therapy'. Animals have been shown to improve patients' emotional, psychological and physical wellbeing. There has however been little research on pet therapy in the outpatient psychiatric population. A 'Pets as therapy' (PAT) dog was present in patient consultations in an adult psychiatry clinic in South Cumbria one day a week during the study period. Patients were given a feedback questionnaire to complete if the PAT dog was present in their consultation. This pilot study was done over a period of seven months from November 2016 to June 2017. Thirty-four patients completed the questionnaire out of $39.100 \%$ of patients enjoyed having the PAT dog present and 91\% felt the PAT dog reduced their anxiety and made them feel more relaxed. $70 \%$ of people felt the PAT dog improved their mood and 88\% would like a PAT dog to be present during consultations in the future. PAT dogs may improve patient experience and, through reducing anxiety, might allow patients to more easily establish a rapport with their psychiatrist which could have positive implications on information they share. More research is required in order to gain a better understanding if there are improved outcomes from using a therapy dog in psychiatry outpatients.

\section{BACKGROUND}

Dogs have been providing companionship for humans for more than 10,000 years. ${ }^{1}$ Unique to dogs is their ability to cooperate and communicate with humans, through their ability to utilise social skills. Humans form attachments to dogs in similar ways they would a child ${ }^{1}$ and many recognise their pet as a part of their central personal network ${ }^{2}$. Pet therapy is therefore becoming increasingly popular as an adjunctive treatment option for those with physical and mental health disorders.

One of the earliest documented uses of pet therapy for psychiatric patients in the UK was in the 1700s when the 'York Retreat' utilized animals. Famous for having pioneered the so-called "moral treatment" that became a model for asylums around the world, they also had several domesticated animals, including rabbits, seagulls, and hawks. Since then pet therapy (also known as animalassisted therapy) in psychiatric inpatient settings has been gathering interest and there have been several research studies that suggest pet therapy can improve patients' emotional, psychological, and physical wellbeing. Recent studies demonstrate several benefits of pet therapy including improved quality of life, ${ }^{3}$ reduced aggression and violence,, 5 and perhaps the most beneficial effect is a reduction in depressive symptoms. ${ }^{6}$ To date these studies have taken place in an inpatient setting only. The National Institute for Health and Care Excellence (NICE) recommend animal-assisted therapy as a treatment option for people with dementia who experience agitation, depression or anxiety. ${ }^{7}$ The touch of an animal has been shown to increase the release of endorphins, allowing the body to relax. ${ }^{8}$

Pet therapy in the outpatient setting is less studied, with most of the literature focussing on children. In the 1960s, a psychiatrist, Dr Levinson, embarked on research of pet therapy with children after he had taken his dog, Jingles, to work. His patient, a non-verbal boy, arrived early for his outpatient appointment and unintentionally met Jingles. The young boy interacted with Jingles in a way he never had with his doctor or family. Dr Levinson realised the benefit of using his dog as a strategy to bridge communication with his young patients.9, 10 Marguerite E. O'Haire et al. also studied the effects of pet therapy on children, specifically those with autistic spectrum disorder, and identified significant improvements in social functioning and a decrease in social withdrawal

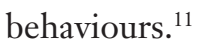

When considering the use of pet therapy, it is important to assess any potential health and safety risks. Important qualities needed by a therapy dog are calmness and accepting of strangers. Therapy dogs are temperament tested by 'Pets as Therapy UK', who provides public liability insurance for dogs who are deemed suitable. The Care Quality Commission has confirmed that animals are allowed onto wards, subject to local policies. ${ }^{12}$ Another factor to consider is the impact on patients with allergies to dogs. It is well documented that people have allergies to dog dander. Given the ubiquity of pet dogs, dog dander occurs in almost all communal areas, especially in places such as schools and doctors surgeries, where a large number of people who have had contacts with dogs at home bring dog dander with them on their clothes. ${ }^{13} \mathrm{~A}$ search of the literature was not able to find any recorded cases of anaphylaxis to dog dander. It is important to ensure the patient is aware that a therapy dog will be present during the consultation and have the opportunity to decline this. This is also especially relevant to patients with fears or cultural disapproval of having a dog present.

\section{AlM}

This study aimed to evaluate the feedback received from patients who experienced the presence of, and interaction with, a Pets as Therapy dog (PAT dog), in a general adult psychiatry outpatient clinic.

\section{METHOD}

The PAT dog was called Luna, a female Lurcher, born approximately in 2009, adopted by Dr Adam Joiner from Dogs Trust Merseyside in January 2016. Luna is registered with the charity Pets as Therapy UK (Registration number: 1106751). She was present during some outpatient clinics with Dr Joiner (consultant adult psychiatrist) in Barrowin-Furness and Millom between November 2016 and June 2017. Patients were given prior knowledge, both written and verbal, of the presence of Luna. Patients had the choice for Luna to be taken out of the clinic room. Patients with 
all presentations and diagnoses were included in this study.

At the end of the consultation Dr Joiner gave patients a feedback questionnaire. Patients were asked to complete it in the waiting room and return it to reception staff to ensure some anonymity. The survey was brief, taking no more than one minute to complete (see appendix 1). The questionnaire consisted of five Likert questions and one open free-text question. Simple descriptive statistics were used to analyse the data, and the free text was searched for themes.

\section{RESULTS}

Thirty four out of 39 patients completed and returned the questionnaire. Four patients declined to complete the survey and one patient asked that Luna was not present. Table 1 shows the responses to the five Likert questions. $100 \%$ of patients who completed the questionnaire enjoyed having Luna present. Patients were keen for Luna to be present in future outpatient appointments, with $53 \%$ strongly agreeing with this and 35\% agreeing; $18 \%$ did not mind either way, and no one disagreed with having Luna present in the future.

From our results, patients found that Luna was more likely to positively impact on their level of anxiety than their mood: $91 \%$ of patients agreed or strongly agreed that Luna reduced their anxiety and made them feel more relaxed (44\% strongly agreed). Two patients $(6 \%)$ neither agreed nor disagreed that Luna reduced anxiety, although one patient (3\%) disagreed. Twenty-four patients $(71 \%)$ agreed or strongly agreed Luna improved their mood, but 10 patients $(29 \%)$ neither agreed nor disagreed that she had an impact on mood.

The presence of Luna was not deemed a distraction for $71 \%$ of patients, with $12 \%$ neither agreeing nor disagreeing. Six patients (17\%) agreed Luna was a distraction. These six patients however answered strongly agree or agree to all other statements and two of these patients specified "this wasn't negative" and "in a positive way". There were no comments which suggested the distraction was negative.

Free text comments were overall positive. The most common theme was of patients expressing gratitude of Luna's presence and commenting on her pleasant manner: "Having a therapy dog present is a good idea"; "She is a lovely dog". Another theme was expanding on positive impact on anxiety: "The addition of the PAT dog in my consultation is a superb idea! She was a lovely distraction that helped ease my anxieties. Highly recommend PAT dogs attending consultations. Thank you!" Others also stated they felt calmer and one patient commented that having Luna present "... made me feel like I was able to open up more". A patient with chronic suicidal thoughts found a therapy dog life-affirming: "Luna is brilliant and inspired me to think of a dog myself which is a positive link to life". The only negative comment was regarding Luna falling asleep "Wish it had not gone asleep in the consultation!!" although this suggests this patient would have preferred more contact with Luna during the consultation.

\section{DISCUSSION}

Luna was very well received in this pilot study; many patients found she had a positive impact on their subjective mental state and others simply enjoyed her being there. This suggests there is an improvement in patient experience for many patients with a therapy dog present. Anxiety symptoms can often impede communication in consultations; it can take time to establish a rapport with some patients with mental illness, which could impact on the quality of care. Having a PAT dog present may allow rapport to be established sooner in the consultation and provide time for the psychiatrist to obtain a more thorough history, although the data here does not allow for this conclusion and it would require further investigation. Although some patients stated Luna was a distraction it is possible this result is due to a variation in the patients' understanding of the statement, as distractions can also be seen as a positive. Two patients commented that the distraction was welcome and positive. In the future this should be more carefully worded, for example to ask if the PAT dog was disruptive. $88 \%$ of patients stated they would like Luna to be in future appointments; there is potential that these patients would be more likely to attend their follow up appointments if they knew Luna would be present. Improving non-attendance at psychiatry outpatients is a long-standing issue. The impact of a therapy dog on attendance at outpatient appointments should be studied in the future.

Observations of how different patients interacted with Luna were of interest and would warrant further study. Some patients ignored her during the entire consultation, while others stroked her during most of the consultation.

\begin{tabular}{|c|c|c|c|c|c|}
\hline Statement & $\begin{array}{c}\text { Strongly } \\
\text { Agree }\end{array}$ & Agree & $\begin{array}{c}\text { Neither agree } \\
\text { or disagree }\end{array}$ & Disagree & $\begin{array}{c}\text { Strongly } \\
\text { disagree }\end{array}$ \\
\hline I enjoyed having the therapy dog present & 23 & 11 & - & - & - \\
\hline $\begin{array}{c}\text { The therapy dog made me feel more relaxed or } \\
\text { reduced anxiety }\end{array}$ & 15 & 16 & 2 & 1 & - \\
\hline The therapy dog was a distraction & - & 6 & 4 & 8 & 16 \\
\hline The therapy dog improved my mood & 10 & 14 & 10 & - & - \\
\hline $\begin{array}{c}\text { If I had a further consultation I would like a } \\
\text { therapy dog to be present }\end{array}$ & 18 & 12 & 4 & - & - \\
\hline
\end{tabular}

Table 1: Responses to PAT dog feedback questionnaire in the repeat study 
Most commonly, patients would interact with her at the beginning and end. Some would stroke her intermittently during the consultation, and this was sometimes when talking about something distressing or difficult (but not always).

From this pilot study, Luna had a more positive impact on anxiety than mood. Use of PAT dogs could be targeted to specific patients if there was a better understanding of who would be more likely to benefit from them. Future studies could assess whether PAT dogs have a variable effect with specific diagnoses, severity of illness and patient presentation by including patient symptoms and/or diagnosis, as well as age and gender. It may also be useful to use objective measures of anxiety and mood (e.g. GAD-7 and PHQ-9), and compare to a control group without a therapy dog in future studies.

Other factors to consider in future studies and limitations of this study include the small sample size. Increasing the time the PAT dog spends in clinic each week will help to address this (although this will need to be balanced with the fatigue that dogs do experience in such environments). Extending the time period of the study will also allow for follow up appointments to be included. This study was limited to a relatively rural location, larger studies could be used to highlight any significant differences between communities, such as urban versus rural.

\section{REFERENCES}

1. Cirulli F, Borgi M, Berry A, Francia N, Alleva E. Animal-assisted interventions as innovative tools for mental health. Ann Ist Super Sanita. 2011;47(4):341348.

2. Brooks H, Rushton K, Walker S, Lovell K, Rogers, A. Ontological security and connectivity provided by pets: a study in the self-management of the everyday lives of people diagnosed with a long-term mental health condition. BMC Psychiatry. 2016;16:409.

3. Schramm E, Hediger K, Lang UE. From animal behavior to human health: an animal-assisted mindfulness intervention for recurrent depression. Zeitschrift für Psychologie. 2015;223(3):192-197.

4. Nurenberg JR, Schleifer SJ, Shaffer TM, et al. Animalassisted therapy with chronic psychiatric inpatients: equine-assisted psychotherapy and aggressive behaviour. Psychiatric Serv. 2015;66(1):80-86.

5. Walsh PG, Martin PG, Verlander DF, et al. The effects of a 'pets as therapy' dog on persons with dementia in a psychiatric ward. Australian Occupational Therapy Journal. 1995;42:161-166.

6. Souter MA, Miller MD. Do animal-assisted activities effectively treat depression? A meta-analysis. Anthrozoos.2007;20(2):167-180.

7. NICE (2006). Dementia: supporting people with dementia and their carers in health and social care [CG42]. Available at: https://www.nice.org.uk/ guidance/cg42. (Accessed on 24.10.17)

8. Fine AH, Handbook on animal-assisted therapy: theoretical foundations and guidelines for practice. 2nd ed. San Diego, CA: Elsevier, 2006, pg. 11.

9. Levinson BM, Human/companion animal therapy. Journal of Contemporary Psychotherapy. 1984;14(2):131-144.
10. Hooker SD, Freeman LH, Stewart P. Pet therapy research: a historical review. Holistic Nurs Pract. 2002;16(5):17-23.

11. O'Haire ME, et al. Effects of classroom animalassisted activities on social functioning in children with autism spectrum disorder. The Journal of Alternative and Complementary Medicine. 2014;20(3):162-168.

12. Animal Magic. Available at: http://www.animalmagic.org.uk/introduction/yes-animals-are-allowed/ (accessed on 24.10.17)

13. Allergy UK, (2017) Allergy to domestic pets. Available at https://www.allergyuk.org/allergy- to-domesticpets/allergy-to-domestic-pets (accessed 24.10.17).

\section{APPENDIX 1}

\section{PAT Dog Feedback Questionnaire}

\section{This survey will take ONE minute to fill out}

Please circle one answer for each question

1) I enjoyed having a therapy dog present during the consultation

Strongly agree agree neither agree or disagree disagree strongly disagree

2) The therapy dog made me feel more relaxed or reduced anxiety

Strongly agree agree neither agree or disagree disagree strongly disagree

3) The therapy dog was a distraction

Strongly agree agree neither agree or disagree disagree strongly disagree

4) The therapy dog improved my mood

Strongly agree agree neither agree or disagree disagree strongly disagree

5) If I had a further consultation I would like a therapy dog to be present

$\begin{array}{ll}\text { Strongly agree agree } & \text { neither agree or } \\ \text { disagree disagree } & \text { strongly disagree }\end{array}$

Any additional comments?

Thank you for taking the time to fill out this survey.

Declaration of Interests: All authors have no interests to declare.

Correspondence to: Adam.Joiner@lancashirecare.nhs.uk Adam Joiner (LCFT), Consultant Psychiatrist 